\title{
Inhibiting function of human fetal dermal mesenchymal stem cells on bioactivities of keloid fibroblasts
}

Ya Jiao ${ }^{1,2 \dagger}$, Xiao Wang ${ }^{1,2 \dagger}$, Jixun Zhang ${ }^{1,2}$, Yongjun Qi ${ }^{1,2}$, Hongmin Gong ${ }^{1,2}$ and Duyin Jiang ${ }^{1,2^{*}}$

\begin{abstract}
Background: Keloid is one kind of benign skin disease caused by hyperplasia of fibroblasts and collagen fibrils. It is refractory due to the lack of an effective treatment at present, which puts pressure on seeking a new therapeutic regimen. Mesenchymal stem cells (MSCs) from fetal skin are considered to play a crucial role in scarless healing. Nevertheless, the efficacy of them in keloid disorders remains poorly understood.

Methods: Keloid fibroblasts (KFs), human adult dermal fibroblasts (ADFs), and human fetal dermal mesenchymal stem cells (FDMSCS) were isolated to single cells and cultured in Dulbecco's modified Eagle's medium (DMEM). ADFs and FDMSCs were used to generate ADF-conditioned medium (A-CM) and FDMSC-conditioned medium (FCM). The effects of A-CM and F-CM on KFs were tested using MTT assay, BrdU assay, TUNEL assay, quantitative polymerase chain reaction, Western blot, and annexin V-FITC/PI binding assay,
\end{abstract}

Results: FDMSCs inhibited the bioactivity of KFs, downregulated the expression of the antiapoptotic protein BCL-2, and upregulated the expression of the proapoptotic protein BAX of KFs by secreting some soluble substances, thus accelerating the apoptosis of KFs.

Conclusion: F-CM induces apoptosis of KFs, providing a novel treatment strategy for keloid disorders.

Keywords: Fetal dermal mesenchymal stem cells, Keloid, Conditioned medium

\section{Background}

As one kind of benign skin disease, keloid is caused by hyperplasia of fibroblasts and collagen fibrils [1]. Keloids can grow excessively and invade nearby healthy skin, so the patient with keloid suffers from great physical and psychological pressure [2]. Although keloid is a clinically frequently occurring disease, it is refractory due to the lack of an effective treatment. Therefore, there is a pressing need to seek a new therapeutic regimen. Recent research has indicated that mesenchymal stem cells (MSCs) play significant roles in scarless wound healing and tissue regeneration [3-5]. In addition, fetal skin cells are thought to play key roles in fetal scarless healing $[6,7]$.

\footnotetext{
*Correspondence: jdybs2@vip.163.com

${ }^{\dagger}$ Equal contributors

'Department of Emergency and Department of Burns and Plastic Surgery,

The Second Hospital of Shandong University, Jinan, Shandong 250033,

People's Republic of China

${ }^{2}$ School of Medicine, Shandong University, Jinan, Shandong 250012, People's Republic of China
}

(c) The Author(s). 2017 Open Access This article is distributed under the terms of the Creative Commons Attribution 4.0 International License (http://creativecommons.org/licenses/by/4.0/), which permits unrestricted use, distribution, and reproduction in any medium, provided you give appropriate credit to the original author(s) and the source, provide a link to the Creative Commons license, and indicate if changes were made. The Creative Commons Public Domain Dedication waiver (http://creativecommons.org/publicdomain/zero/1.0/) applies to the data made available in this article, unless otherwise stated.

\section{Results}

$\mathrm{F}-\mathrm{CM}$ induces the apoptosis of KFs

As we know, morphology is the most direct representation of the cell state. Cells with good growth status have excellent transparency, high refraction, and a clear boundary. On the contrary, cells in bad condition have inferior transparency, low refraction, and an unclear boundary, and even lose the characteristics of the original cells. As a control group, KFs cultured with serum-free medium (SFM) showed spindle or 
triangular shapes with a clear boundary, which had excellent transparency and high refraction. KFs cultured with adult dermal fibroblast (ADF)-conditioned medium (A-CM) looked similar to the control group with high cell density and an orderly arrangement (Fig. 1a). As for the F-CM group, we observed a decreased cell density, increased number of floating cells, and the characteristic apoptotic appearance including cell shrinkage, chromatin condensation, membrane blebbing, formation of apoptotic bodies, and disordered cell arrangement [8] (Fig. 1a). Meanwhile, the number of living KFs in the F-CM group was much less than in the control group, which was not embodied in the A-CM group (Fig. 1b). Furthermore, we detected the effects of F$\mathrm{CM}$ and $\mathrm{A}-\mathrm{CM}$ on the proliferation and apoptosis of KFs; neither A-CM nor F-CM had any significant effect on KF proliferation (Fig. 1c). However, the percentage of TUNELpositive cells in the F-CM group was $22.24 \pm 2.05 \%$, much higher than in the control group and A-CM group (Fig. 1d), indicating that F-CM promoted the apoptosis of KFs. Taken together, these data suggest that F-CM inhibits the bioactivity of KFs by mainly inducing cell apoptosis.

\section{F-CM effects the expression of BCL-2 and BAX}

It is well known that the antiapoptotic protein BCL-2 inhibits cell apoptosis by blocking cytochrome $\mathrm{C}$ release from mitochondria. In various kinds of tumor cells, the expression of BCL-2 is upregulated $[9,10]$. The proapoptotic protein BAX could form heterodimers with BCL-2, and suppress the antiapoptotic effect of BCL-2. As a result, the upregulation of BCL-2 promotes apoptosis [11]. The ratio of BCL-2 expression to BAX expression is a direct index of cell apoptosis. To investigate the molecular mechanism of $\mathrm{F}-\mathrm{CM}$ on KFs, quantitative real-time polymerase chain reaction (qPCR) and Western blot were performed.

As shown in Fig. 2a, the mRNA level of $\mathrm{Bcl}-2$ was downregulated in the F-CM group while the A-CM group showed no significant difference compared with the control group. Meanwhile, the RNA level of Bax was upregulated in the F-CM group while the A-CM group showed only a very slight change compared with the control group (Fig. 2b). As a result, the $B c l-2 / B a x$ ratio of the F-CM group was much lower than the control group (Fig. 2c), which indicates that the F-CM group has more apoptotic cells. Interestingly, the $B c l-2 / B a x$ ratio of the A-CM group was a little lower than that of the control group. We suspected that ADFs absorbed some nutrients and released some metabolic waste into the A$\mathrm{CM}$, which also happened in the experimental study of others [12]; the cell medium must be replaced regularly to avoid affecting the survival status of cells. Consistently, the protein level of BCL-2 was downregulated, and the protein level of BAX was upregulated in the F$\mathrm{CM}$ group. However, there was no significant change in $\mathrm{BCL}-2$ and BAX protein levels in the A-CM group (Fig. 2d), which was inconsistent with the mRNA levels. Translation of individual mRNA species into their encoded proteins is regulated producing discrepancies between mRNA and protein levels, which may resulted

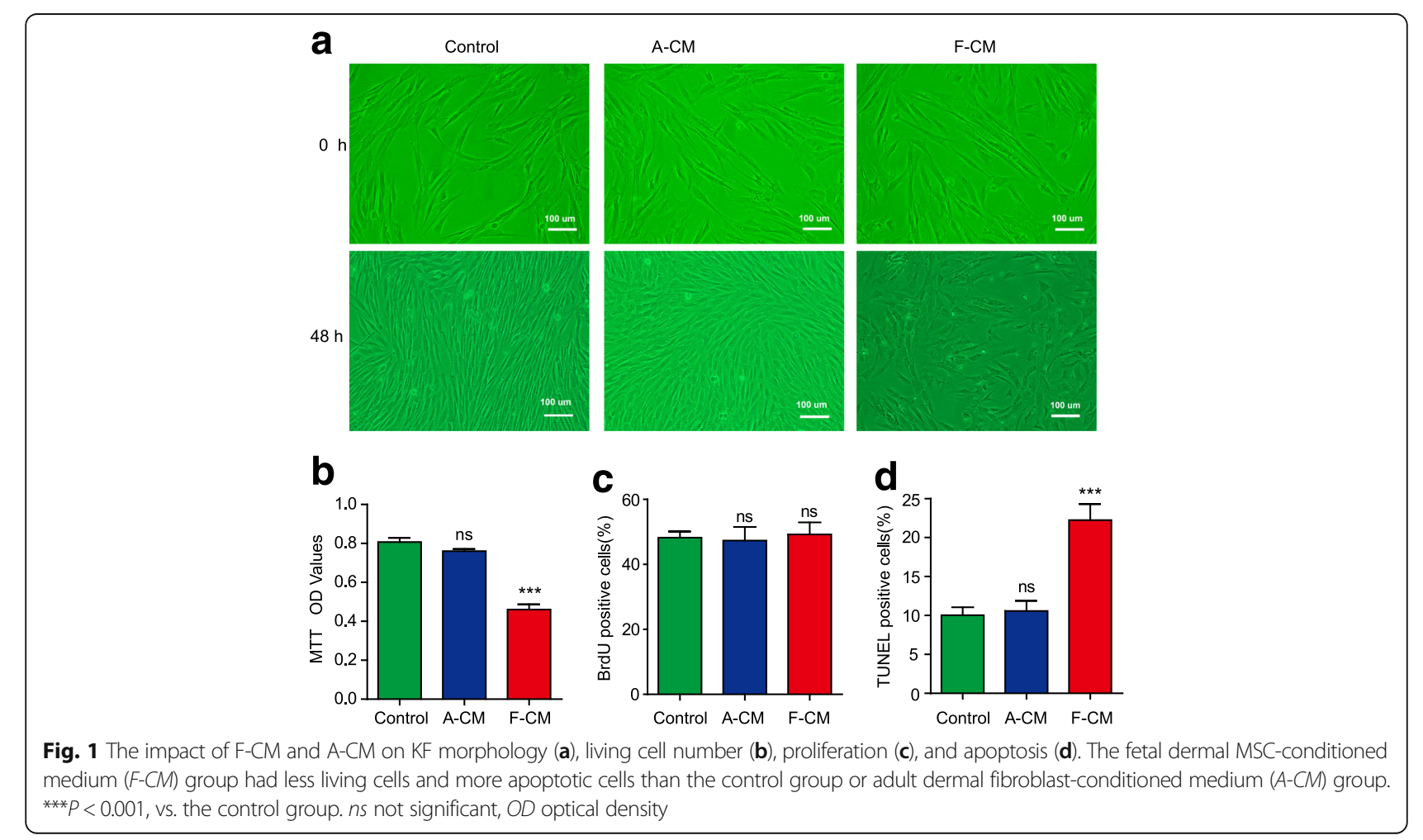



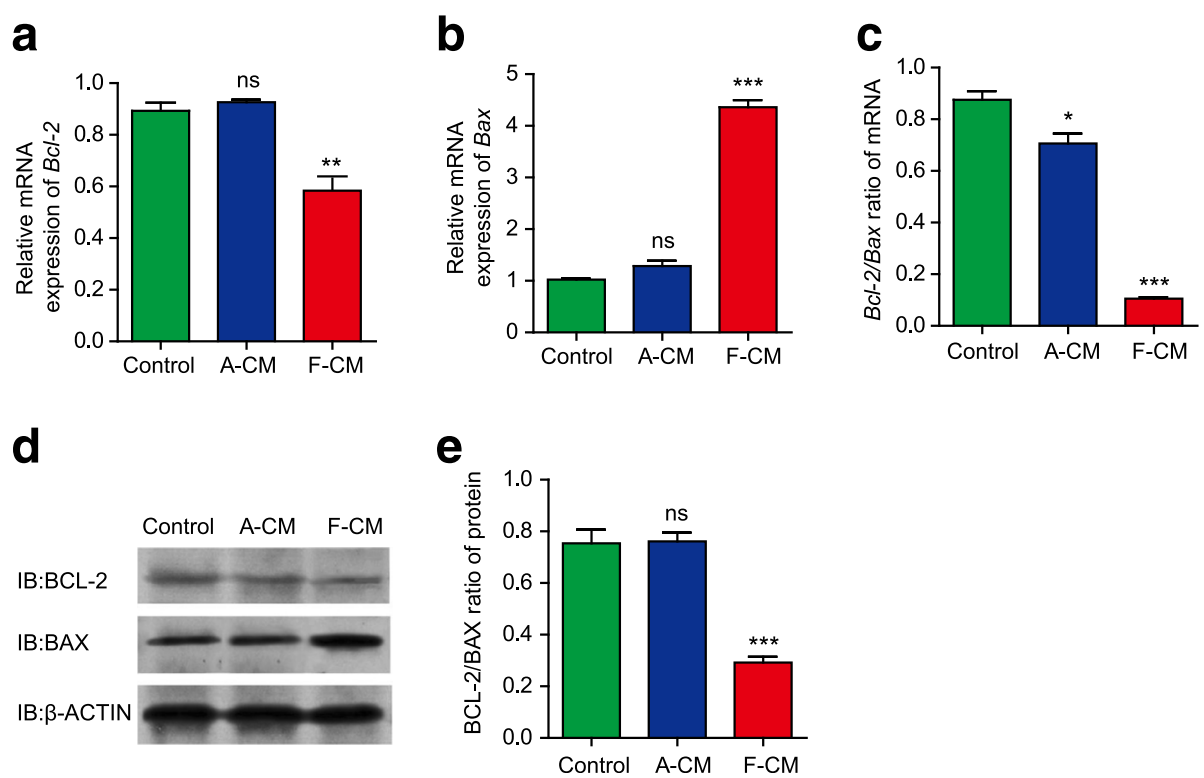

Fig. 2 The expression of apoptosis-associated genes and proteins analyzed with qPCR (a-c) and Western blot (d,e). has the effect of promoting the expression of proapoptotic genes and proteins and inhibiting expression of antiapoptotic genes and proteins. ${ }^{*} P<0.05,{ }^{* *} P<0.01,{ }^{* *} P<0.001$, vs. control group. A-CM adult dermal fibroblast-conditioned medium, ns not significant

from altered translational efficiencies [13, 14]. Thus, the BCL-2/BAX ratio of the F-CM group was notably downregulated (Fig. 2e). To summarize, F-CM downregulates BCL-2 expression and upregulates BAX expression of KFs, resulting in KF apoptosis.

\section{F-CM accelerates the late phase of KF apoptosis}

To further investigate the impact of F-CM on KFs, an Annexin V-fluorescein isothiocyanate (FITC)/propidium iodide (PI) binding assay was performed. In living cells the cell membrane is impermeable to V-FITC and PI. In early apoptotic cells, phosphotidylserine is translocated to the extracellular surface of the cell membrane. Annexin V-FITC specifically binds with phosphotidylserine. However, the cell membrane of early apoptotic cells is still impermeable to PI. In late apoptotic cells, the cell membrane is ruptured and permeable to V-FITC and PI. In dead cells the cell membrane is destroyed completely and stained with PI only [15]. We could distinguish and quantitatively determine the percentage of dead cells (Annexin VFITC-negative/PI-positive), viable cells (Annexin V-FITCnegative/PI-negative), early apoptotic cells (Annexin VFITC-positive/PI-negative), and late apoptotic cells (Annexin V-FITC-positive/PI-positive).

Our research revealed that there were more apoptotic cells in the F-CM group than the A-CM group and the control group (Fig. 3a). Moreover, the proportion of late apoptotic cells was significantly increased as shown in Fig. 3b, indicating that F-CM mainly induced the late phase of apoptosis.

\section{Discussion}

The etiology of keloid is unknown, and the complexity of its development without specific factors being identified has caused difficulties in finding effective treatment. The main histopathologic features of keloid are extracellular matrix (ECM) degradation and collagen remodeling. These processes are regulated by the matrix metalloproteinases (MMPs) with significantly elevated activity and increased expression in KFs [16]. MicroRNA-205-5p of KFs, which is known to induce cell apoptosis, inhibit cell invasion and migration, and impair cell viability, is downregulated in KFs [17]. In addition, many reports have demonstrated that KFs, as one of the primary research targets, were the key cellular mediators of fibrogenesis. The role of KFs in keloid is attracting particular attention for a therapeutic effect.

In addition to some traditional therapies, there are many new therapeutic approaches, such as stem cell therapy, that have brought new hopes to managing keloid. Recent studies show that MSCs can regulate the wound-healing process and prevent scar formation in keloid formation, suggesting their promising therapeutic role in keloid. The paracrine role of MSCs is also attracting particular attention for their therapeutic effect on wound healing, tissue repair, and scar remodeling [18-20]. Reports have demonstrated that the use of adipose-derived MSCs could facilitate cutaneous wound healing and form a thinner scar, and the underlying mechanisms involved are the downregulated expression of transforming growth factor (TGF)- $\beta 1$, decreased accumulation of collagen, and increased expression of 

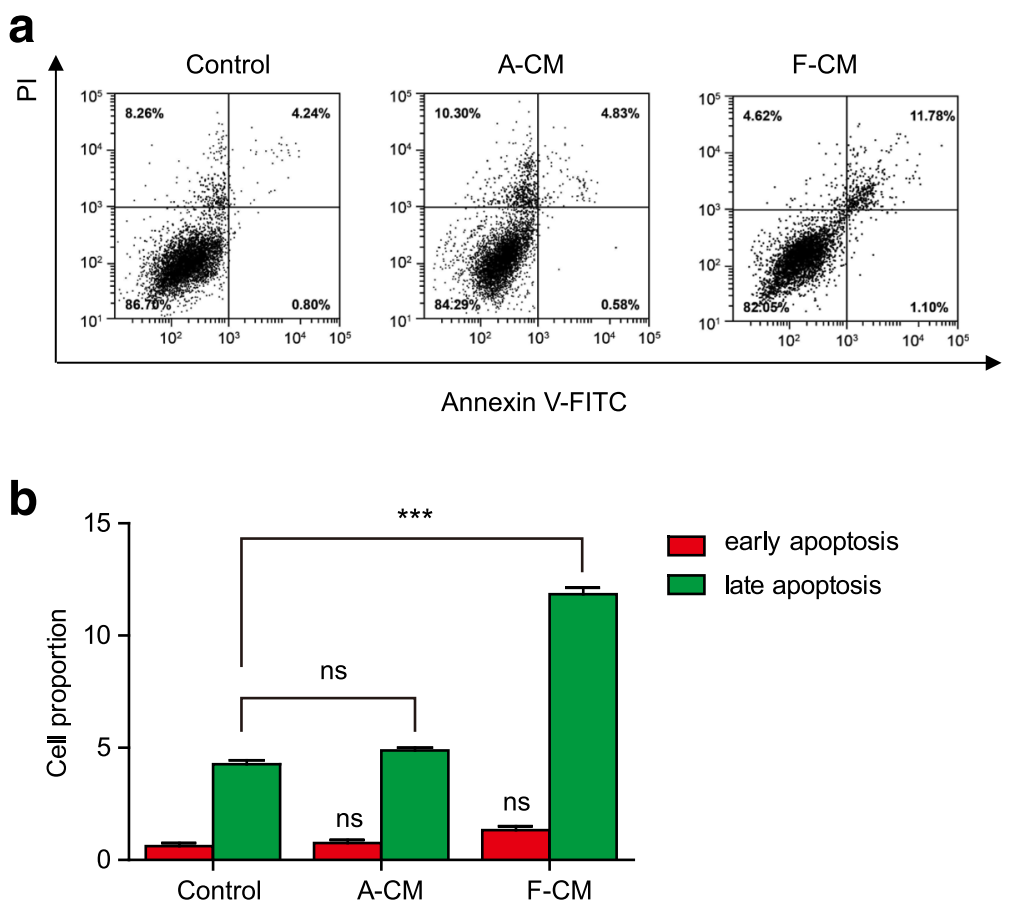

Fig. 3 Flow cytometry analysis of KFs. a Scatter plots of fluorescein isothiocyanate (FITC)-fluorescence versus propidium iodide (PI)-fluorescence in the three groups. $\mathbf{b}$ The proportion of early and late apoptosis cells in the three groups, which implied that F-CM could mainly induce late apoptosis of KFs. ${ }^{* *} P<0.001$, vs. control group. A-CM adult dermal fibroblast-conditioned medium, ns not significant

decorin [21]. Bone marrow-derived MSCs pathologically suppressed excessive skin fibrosis, inhibited KF proliferation, and ECM deposition through TGF- $33-$ dependent activation, and adult tissue-derived MSCs are candidates for cellular therapy to promote scarless wound healing by attenuating inflammation [22]. Furthermore, MSC transplantation therapy has shown safety in effectively promoting skin wound healing in recent years.

In contrast to the adult, fetal wound healing can happen and repair skin wounds with the absence of scar formation in the early and middle embryo stage of mammals, which provides a potential therapeutic implication for human adult wounds. Although, the exact mechanisms of scarless fetal wound healing remain largely unknown, they are thought to be mainly due to the special properties of the embryo [23, 24]. Fetal wound healing is characterized by the attenuated inflammation with fewer proinflammatory cytokines, and the fetal ECM being rich in type III collagen and hyaluronan plays an important role in scarless wound healing as well [25]. Moreover, other differences between fetal skin cells and postnatal skin cells, such as gene expression, cellular mediators, and stem cell function, contribute to the different outcomes [26, 27].

Based on the research results above, we subsequently selected the intersection of MSCs and fetal skin cells as our breakthrough point: FDMSCs. We hypothesized that FDMSCs could regulate the bioactivity of KFs. To verify our hypothesis, we designed experiments to demonstrate the function of FDMSCs. KFs were incubated with ACM, F-CM, and SFM. We found that F-CM significantly induces cell apoptosis of KFs. Consistent with the effect of F-CM, coculture of FMSCs and KFs also induced the apoptosis of KFs (Additional file 1: Figure S1). Stem cell transplantation is not at our disposal now owing to undiscovered characteristics of stem cells. Application of the conditioned medium is a better choice.

FDMSCs show superiority, including low immunogenicity, easy expansion in vitro, and higher proliferation potential and differentiation capability compared with stem cells from other sources. FDMSCs were not recognized by $\mathrm{T}$ lymphocytes after transplantation and immunological rejection was avoided. Therefore, FDMSCs show significant promise for their clinical application for safety and effectiveness [28]. In order to further verify the safety of F$\mathrm{CM}$, we have performed BrdU double staining with MSC markers to detect whether renewing KFs changed their nature. The results showed that BRDU-positive cells were negative for MSCs markers (Additional file 1: Figure S9). The renewing KFs were not transdifferentiated under F$\mathrm{CM}$ conditions, which provides further evidence of its safety. In addition, time-dependent effects of F-CM were observed which showed that the ratio of cell death rose in a time-dependent manner; just like with clinical drugs, clinical treatment needs appropriate concentration, administration time, and duration. 


\section{Conclusion}

Our research revealed that FDMSCs downregulated the expression of the antiapoptotic protein BCL-2 and upregulated the expression of the proapoptotic protein BAX of KFs by secreting soluble substances. Therefore, FDMSCs accelerates the apoptosis of KFs, providing novel treatment strategies for keloid.

\section{Methods}

\section{Tissue procurement}

All tissues were obtained from patients of The Second Hospital of Shandong University. Fetal skin tissues were taken from 16-20 week human fetuses which were accidental abortions without hereditary disease or drug therapy. Adult skin tissues were obtained from patients who underwent cosmetic surgery (surgical waste). Keloid tissues were taken from keloid patients confirmed by pathology without any treatment in the previous 3 years. There were five specimens for each sample at least, and all samples were stored at $4{ }^{\circ} \mathrm{C}$ in phosphate-buffered saline (PBS; Invitrogen, USA) containing $100 \mathrm{U} / \mathrm{ml}$ penicillinstreptomycin (Gibco, USA).

\section{Cell isolation and cell culture}

Fetal skin tissues from the dorsum were carefully dissected into $1.0-\mathrm{cm}^{2}$ pieces and digested with $0.25 \%$ dispase (Sigma, USA) overnight at $4{ }^{\circ} \mathrm{C}$ to remove the epidermis completely. Tissues were then cut into 0.1$\mathrm{cm}^{2}$ pieces and digested with $0.125 \%$ type I collagenase (Worthington, USA) for $5 \mathrm{~min}$ at $37{ }^{\circ} \mathrm{C}$ to obtain FDMSCs. The adult skin tissues were cut into $1.0-\mathrm{cm}^{2}$ pieces and digested with $0.25 \%$ dispase overnight at $4{ }^{\circ} \mathrm{C}$. Tissues were then cut into $0.1-\mathrm{cm}^{2}$ pieces and digested with $0.125 \%$ type I collagenase for $30 \mathrm{~min}$ at $37{ }^{\circ} \mathrm{C}$ to obtain ADFs. The isolated cells were cultured in lowglucose Dulbecco's modified Eagle's medium (DMEM; HyClone, USA) containing10\% fetal bovine serum (FBS; Gibco, USA) and $1 \% 100 \mathrm{U} / \mathrm{ml}$ penicillin-streptomycin. Keloid tissues without epidermis were minced into pieces as small as possible and then placed in the culture dishes. Two hours later, the minced keloid tissues were cultured in low-glucose DMEM with 10\% FBS and 1\% $100 \mathrm{U} / \mathrm{ml}$ penicillin-streptomycin.

FDMSCs and ADFs attached to the bottom of the dish $12 \mathrm{~h}$ later. After 1 or 2 weeks, KFs migrated from the tissue pieces. All of the cells were cultured at $37^{\circ} \mathrm{C}$ with $5 \% \mathrm{CO}_{2}$. When cells reached $70-80 \%$ confluence, they were digested with $0.25 \%$ trypsin (Sigma, USA) and subcultured. Only 4-6 generations of cells were used for experiments. These cells had no obvious difference in appearance and exhibited a spindle or triangular shape like fibroblasts. When cells reached $90 \%$ confluence they exhibited an interesting morphology, like vorticity or fish school (Additional file 1: Figure S2).

\section{Detection of surface antigens}

The slides of cells were cultured in six-well plates (Corning, USA), fixed, permeabilized, and then immunostained with primary antibodies overnight at $4{ }^{\circ} \mathrm{C}$. Cell slides were then incubated with species-specific fluorochrome-conjugated secondary antibodies (Cell Signaling Technology, USA) for $1 \mathrm{~h}$ at room temperature and shielded from light. Finally, the images were obtained by fluorescence microscopy coupled with a camera (Nikon Eclipse TS100, Japan) and NIS-Elements software (Nikon, Japan). The MSC phenotypes of FDMSCs were characterized by flow cytometry analysis.

The antibodies anti-CD44, anti-CD90, anti-CD105, anti-CD14, anti-CD34, anti-CD45, anti-SSEA-4, antiOCT-4, anti-vimentin, and anti-CK19 were purchased from Cell Signaling Technology, USA.

FDMSCs were positive for MSC markers including CD44, CD90, and CD105 (Additional file 1: Figure S3 and Figure S5) but negative for hematopoietic stem cell markers including CD14, CD34, and CD45 (Additional file 1: Figure S4 and Figure S5). Interestingly, FDMSCs expressed embryonic markers including SSEA-4 and OCT-4 (Additional file 1: Figure S6). All cells were positive for the dermal cell marker vimentin (Additional file 1: Figure S7) and negative for the epidermal cell marker CK19 (Additional file 1: Figure S8).

\section{Multilineage differentiation potential of FDMSCs}

FDMSCs were plated into six-well plates and cultured with osteogenic differentiation medium (Cyagen, China) when cells reached $70 \%$ confluence. The culture medium was changed every 3 days. After 5-7 days, calcium nodules appeared. After 20 days of differentiation, the cells were stained with alizarin red (Cyagen, China). As shown in Additional file 1 (Figure S10), the ECM was rich in calcium, confirming osteogenic differentiation.

FDMSCs were plated into six-well plate and cultured in medium. When cells reached $100 \%$ confluence, the medium was changed to adipogenic differentiation medium (Cyagen, China). After 3 days, the medium was replaced with adipogenic maintenance medium (Cyagen, China) and, $24 \mathrm{~h}$ later, the medium was replaced with adipogenic differentiation medium again. Five to seven days later, the intracellular lipid vacuoles appeared. After four cycles, the cells were stained with Oil-red $\mathrm{O}$ (Cyagen, China). As shown in Additional file 1 (Figure S10), more than $70 \%$ of the cells were positive for Oilred $\mathrm{O}$, confirming adipogenic differentiation.

FDMSCs were plated into six-well plate and cultured in medium. When cells reached $70 \%$ confluence, the medium was changed to chondrogenic differentiation medium (Cyagen, China). Under the induction of chondrogenic differentiation medium, the morphology of cells were changed gradually from the vorticity pattern 
to a chaotic pattern and lost polarity. After about 20 days, the cellular layer shrank and differentiated into adipocytes which could be stained with Alcian blue (Cyagen, China), confirming their chondrogenic phenotype as shown in Additional file 1 (Figure S10).

\section{Preparation of conditioned medium}

When FDMSCs and ADFs reached $80 \%$ confluence in 12-well plates (Corning, USA), cells were cultured in serum-free medium (SFM) overnight. Then cells were cultured with $1 \mathrm{ml}$ SFM per well. F-CM and A-CM were harvested after $48 \mathrm{~h}$ incubation. The conditioned medium was then filter-sterilized through a $0.22-\mu \mathrm{m}$ Millex-GP syringe filter (Millipore, USA) and stored at $80{ }^{\circ} \mathrm{C}$.

\section{Morphology of KFs}

KFs were seeded into a six-well plate at $2 \times 10^{4}$ cells/well density and grown in SFM for $24 \mathrm{~h}$. SFM was replaced by A-CM or F-CM as compared with the control group (SFM group), and the cells were cultured for $48 \mathrm{~h}$ before being observed by inverted microscope.

\section{MTT assay}

Cells were seeded in 96-well plates (Corning, USA) at a density of $3 \times 10^{3}$ cells/well and cultured in SFM for $24 \mathrm{~h}$, and then incubated with F-CM, A-CM, or SFM separately for $48 \mathrm{~h}$ at $37{ }^{\circ} \mathrm{C}$ in a $5 \% \mathrm{CO}_{2}$ humidified atmosphere. 3-(4,5-dimethyl-2-thiazolyl)-2,5-diphenyl-2$\mathrm{H}$-tetrazolium bromide (MTT; Amresco, USA) solution was added to each well to a final concentration of $0.5 \mathrm{mg} / \mathrm{ml}$. MTT is reduced to purple formazan in living cells which can be solubilized with dimethyl sulfoxide (DMSO; Solarbio, China). The optical density (OD) for each well was measured at reference wavelength of $492 \mathrm{~nm}$ using a microplate reader (Thermo Fisher Scientific, USA) to evaluate cell viability.

\section{BrdU assay}

Cells were plated in six-well plates at a concentration of $2 \times 10^{3}$ cells/well. After incubation with F-CM, A-CM, or SFM for 48 h, 5-bromo-2-deoxyuridine (BrdU; SCBT, USA) was added to the medium to a final concentration of $10 \mu \mathrm{mol} / \mathrm{ml}$. Two hours later, KFs were stained with BrdU and DAPI, according to the directions of the BrdU immunofluorescence kit (SCBT, USA). For each group, five random fields were counted to calculate the percentage of BrdU and DAPI double-positive cells.

In order to verify if renewing KFs were induced into MSCs by F-CM, BrdU double staining with MSCs markers including CD44, CD90, and CD109 was performed.

\section{TUNEL assay}

KFs were incubated with TUNEL (Beyotime, China) for $1 \mathrm{~h}$ at $37^{\circ} \mathrm{C}$ followed by DAPI for the identification of nuclei. For each group, five random fields were counted to calculate the percentage of TUNEL and DAPI double-positive cells.

\section{Transwell coculture assay}

In order to observe the direct effects of FDMSCs on KFs, transwell coculture of FDMSCs with KFs was performed. FDMSCs were seeded into the upper chamber while KFs were seeded into the lower chamber. After FDMSCs and KFs were separately cultured in transwell coculture system with $0.4-\mu \mathrm{m}$ pore size inserts (Corning, USA) over $48 \mathrm{~h}$, KFs were prepared for the following experiments including MTT assay, BrdU cell proliferation assay, and TUNEL apoptosis assay.

\section{Quantitative real-time polymerase chain reaction}

Total RNAs were extracted from KFs using Trizol Reagent (Invitrogen, CA, USA) and reverse-transcribed using a SuperScript III First-Strand Synthesis Super Mix Kit (Invitrogen). The primers used in the qPCR to detect the mRNA levels are as follows: 1) forward primer 5'TGGATGACTGAGTACCTGAACCG-3' and reverse primer 5'-TGAGCAGAGTCTTCAGAGACAGC-3' for human B-cell lymphoma-2 (Bcl-2; BGI, China). 2) forward primer 5'-ACTGGACAGTAACATGGAGCTG-3' and reverse primer 5'AGCCCATGATGGTTCTGATCAG-3' for human Bcl2-associated X gene (Bax; BGI, China). and 3) forward primer 5'- GGCACCCAGCACAATGAAG -3' and reverse primer 5'-GCCGATCCACACGGAGTACT-3' for human $\beta$-Actin (BGI, China).

\section{Western blot}

Cells were lysed in RIPA lysis buffer (Beyotime, China) at $4{ }^{\circ} \mathrm{C}$ for $30 \mathrm{~min}$. Protein extracts were applied to $10 \%$ SDS-polyacrylamide gel electrophoresis (SDS-PAGE) and transferred to a nitrocellulose membrane (Bio-Rad, USA). The membranes were incubated with primary antibodies against BCL-2 (1:1000 dilution; Cell Signaling Technology, USA), BAX (1:1000 dilution; Cell Signaling Technology), or $\beta$-actin (1:1000 dilution; Cell Signaling Technology) overnight at $4{ }^{\circ} \mathrm{C}$ and then with secondary antibodies coupled to horseradish peroxidase (HRP; 1:3000 dilution; Cell Signaling Technology) for $1 \mathrm{~h}$ at room temperature. Band signals were detected with an enhanced chemiluminescence (ECL) system (Thermo Fisher Scientific, USA) and then visualized.

\section{Annexin V-FITC/PI binding assay}

According to the instructions of the annexin V-FITC/PI flow cytometric assay kit (Invitrogen, USA), KFs were harvested, washed, and suspended at a density of $1.0 \times$ 
$10^{6}$ cells $/ \mathrm{ml}$ with binding buffer containing FITCconjugated annexin V and PI. The cells were then mixed and incubated at room temperature for $15 \mathrm{~min}$. We obtained and analyzed the scatter plots of FITC fluorescence versus PI fluorescence by flow cytometry.

\section{Statistical analysis}

All of the data were analyzed by Student $t$ test (unpaired) by Statistical Product and Service Solutions (SPSS, IBM Corp., USA). The data are shown as the mean \pm standard deviation (SD) from at least three independent repeated experiments.

\section{Additional file}

\begin{abstract}
Additional file 1: Figure S1. Direct effect of ADFs and FDMSCs on KFs. ***:P<0.001, ns: no significance. Figure S2. Morphology and characterization of KFs, ADFs, and FDMSCs. Figure S3.

Immunofluorescent staining showed that FDMSCs were positive for the mesenchymal stem cell markers CD44, CD90, and CD105. Figure S4. Immunofluorescent staining showed that FDMSCs were negative for the hematopoietic stem cell markers CD14, CD34, and CD45. Figure S5. Flow cytometry showed that FDMSCs were positive for the mesenchymal stem cell markers CD44, CD90, and CD105 (A, B, C) and negative for the hematopoietic stem cell markers CD14, CD34, and CD45 (D, E, F). Figure S6. FDMSCs expressed embryonic markers including SSEA-4 and OCT-4. Figure S7. Immunofluorescent staining showed that KFs, ADFs, and FDMSCs were positive for the dermal cell marker vimentin. Figure S8. Immunofluorescent staining showed that KFs, ADFs, and FDMSCs were negative for the epidermal cell marker CK19. Figure S9. Immunofluorescent staining and BrdU staining showed that F-CM did not

alter the nature of KFs. Figure S10. Multilineage differentiation potential of FDMSCs. Alizarin red staining for osteocytes, Oil-red O staining for adipocytes, and Alcian blue staining for Chondrocytes. (DOCX $1210 \mathrm{~kb}$ )
\end{abstract}

\section{Abbreviations}

A-CM: Adult dermal fibroblast-conditioned medium; ADF: Adult dermal fibroblast; Bax: Bcl2-associated X; Bcl-2: B-cell lymphoma-2; BrdU: 5-Bromo-2deoxyuridine; DMEM: Dulbecco's modified Eagle's medium; DMSO: Dimethyl sulfoxide; ECM: Extracellular matrix; FBS: Fetal bovine serum; F-CM: Fetal dermal mesenchymal stem cell-conditioned medium; FDMSC: Fetal dermal mesenchymal stem cell; FITC: Fluorescein isothiocyanate; KF: Keloid fibroblast; MSC: Mesenchymal stem cell; MTT: 3-(4,5-dimethyl-2-thiazolyl)-2,5-diphenyl-2H-tetrazolium bromide; OD: Optical density; PI: Propidium iodide; QPCR: Quantitative real-time polymerase chain reaction; SD: Standard deviation; SFM: Serum-free medium; TGF: Transforming growth factor

\section{Acknowledgements}

We thank the members of the central laboratory for support on flow cytometry of FDMSCs.

\section{Funding}

This research was supported by The National Natural Science Foundation of China (grant Nos. 30772258, 81071560, and 81372074), the High-Tech Research and Development Program of Shandong Province (grant No. 2009GG10002078), and the Science and Technology Development Projects of Shandong province (grant No. 2015GSF118041).

\section{Availability of data and materials}

Supporting data can be obtained from the corresponding author.

\section{Authors' contributions}

YJ and XW performed all experiments and analyzed data. JZ, YQ, and HG performed the cell culture. DJ conceived and supervised the study and wrote the paper. All authors read and approved the final manuscript.

\section{Ethics approval and consent to participate}

The certificate was issued by the Ethics Review Committee of The Second Hospital of Shandong University. The certificate number is KYLL-2017(p)0001. We also received the informed consent of patients.

\section{Consent for publication}

All authors declare their support for the publication and its contents.

\section{Competing interests}

The authors declare that they have no competing interests.

\section{Publisher's Note}

Springer Nature remains neutral with regard to jurisdictional claims in published maps and institutional affiliations.

Received: 9 March 2017 Revised: 28 April 2017

Accepted: 29 June 2017 Published online: 18 July 2017

\section{References}

1. Tredget EE, Levi B, Donelan MB. Biology and principles of scar management and burn reconstruction. Surg Clin North Am. 2014;94(4):793-815.

2. Pitche P. What is true in "spontaneous" keloids? Ann Dermatol Venereol. 2006;133(5 Pt 1):501

3. Lee MJ, Jung J, Na KH, et al. Anti-fibrotic effect of chorionic plate-derived mesenchymal stem cells isolated from human placenta in a rat model of CCI(4)-injured liver: potential application to the treatment of hepatic diseases. J Cell Biochem. 2010;111(6):1453-63.

4. Jackson WM, Nesti $\amalg$, Tuan RS. Mesenchymal stem cell therapy for attenuation of scar formation during wound healing. Stem Cell Res Ther. 2012;3(3):20

5. Teng $M$, Huang $Y$, Zhang $H$. Application of stems cells in wound healing — an update. Wound Repair Regen. 2014;22(2):151-60.

6. Bielefeld KA, Amini-Nik S, Alman BA. Cutaneous wound healing: recruiting developmental pathways for regeneration. Cell Mol Life Sci. 2013;70(12): 2059-81.

7. Hu MS, Rennert RC, Mcardle A, et al. The role of stem cells during scarless skin wound healing. Adv Wound Care (New Rochelle). 2014;3(4):304-14.

8. Singh N, Khanna N, Sharma H, et al. Insights into the molecular mechanism of apoptosis induced by TNF-alpha in mouse epidermal JB6-derived RT-101 cells. Biochem Biophys Res Commun. 2002;295(1):24-30.

9. Park HJ, Jeon YK, You DH, et al. Daidzein causes cytochrome c-mediated apoptosis via the Bcl-2 family in human hepatic cancer cells. Food Chem Toxicol. 2013;60:542-9.

10. Lin HI, Lee YJ, Chen BF, et al. Involvement of Bcl-2 family, cytochrome $\mathrm{c}$ and caspase 3 in induction of apoptosis by beauvericin in human non-small cell lung cancer cells. Cancer Lett. 2005:230(2):248-59.

11. Fickova M, Macho L, Brtko J. A comparison of the effects of tributyltin chloride and triphenyltin chloride on cell proliferation, proapoptotic p53, Bax, and antiapoptotic BCl-2 protein levels in human breast cancer MCF-7 cell line. Toxicol In Vitro. 2015:29(4):727-31.

12. Funayama E, Chodon T, Oyama A, et al. Keratinocytes promote proliferation and inhibit apoptosis of the underlying fibroblasts: an important role in the pathogenesis of keloid. J Invest Dermatol. 2003;121(6):1326-31.

13. Gygi SP, Rochon $Y$, Franza BR, et al. Correlation between protein and mRNA abundance in yeast. Mol Cell Biol. 1999;19(3):1720-30.

14. Mackay VL, Li X, Flory MR, et al. Gene expression analyzed by highresolution state array analysis and quantitative proteomics: response of yeast to mating pheromone. Mol Cell Proteomics. 2004;3(5):478-89.

15. Sharma V, Anderson D, Dhawan A. Zinc oxide nanoparticles induce oxidative DNA damage and ROS-triggered mitochondria mediated apoptosis in human liver cells (HepG2). Apoptosis. 2012:17(8):852-70.

16. Imaizumi $R$, Akasaka $Y$, Inomata $N$, et al. Promoted activation of matrix metalloproteinase (MMP)-2 in keloid fibroblasts and increased expression of MMP-2 in collagen bundle regions: implications for mechanisms of keloid progression. Histopathology. 2009;54(6):722-30.

17. An $G$, Liang $S$, Sheng $C$, et al. Upregulation of microRNA-205 suppresses vascular endothelial growth factor expression-mediated PI3K/Akt signaling transduction in human keloid fibroblasts. Exp Biol Med (Maywood). 2017; 242(3):275-85. 
18. Kamolz LP, Keck M, Kasper C. Wharton's jelly mesenchymal stem cells promote wound healing and tissue regeneration. Stem Cell Res Ther. 2014; 5(3):62.

19. Arno Al, Amini-Nik S, Blit PH, et al. Effect of human Wharton's jelly mesenchymal stem cell paracrine signaling on keloid fibroblasts. Stem Cells Transl Med. 2014;3(3):299-307.

20. Fong CY, Biswas A, Subramanian A, et al. Human keloid cell characterization and inhibition of growth with human Wharton's jelly stem cell extracts. J Cell Biochem. 2014;115(5):826-38.

21. Lee SH, Lee JH, Cho KH. Effects of human adipose-derived stem cells on cutaneous wound healing in nude mice. Ann Dermatol. 2011;23(2):150-5.

22. Wu Y, Peng Y, Gao D, et al. Mesenchymal stem cells suppress fibroblast proliferation and reduce skin fibrosis through a TGF-beta3-dependent activation. Int J Low Extrem Wounds. 2015;14(1):50-62.

23. Namazi MR, Fallahzadeh MK, Schwartz RA. Strategies for prevention of scars: what can we learn from fetal skin? Int J Dermatol. 2011;50(1):85-93.

24. Larson BJ, Longaker MT, Lorenz HP. Scarless fetal wound healing: a basic science review. Plast Reconstr Surg. 2010;126(4):1172-80.

25. Leung A, Crombleholme TM, Keswani SG. Fetal wound healing: implications for minimal scar formation. Curr Opin Pediatr. 2012;24(3):371-8.

26. Wulff BC, Yu L, Parent AE, et al. Novel differences in the expression of inflammation-associated genes between mid-and late-gestational dermal fibroblasts. Wound Repair Regen. 2013;21(1):103-12.

27. White $\mathrm{P}$, Thomas DW, Fong $\mathrm{S}$, et al. Deletion of the homeobox gene PRX-2 affects fetal but not adult fibroblast wound healing responses. J Invest Dermatol. 2003;120(1):135-44

28. Hohlfeld J, de Buys RA, Hirt-Burri N, et al. Tissue engineered fetal skin constructs for paediatric burns. Lancet. 2005:366(9488):840-2.

\section{Submit your next manuscript to BioMed Central and we will help you at every step:}

- We accept pre-submission inquiries

- Our selector tool helps you to find the most relevant journal

- We provide round the clock customer support

- Convenient online submission

- Thorough peer review

- Inclusion in PubMed and all major indexing services

- Maximum visibility for your research

Submit your manuscript at www.biomedcentral.com/submit 